\title{
A note on kernel density estimation at a parametric rate
}

\author{
J. E. Chacón, J. Montanero, A. G. Nogales \\ Dpto. de Matemáticas, Universidad de Extremadura, SPAIN.
}

\begin{abstract}
In the context of kernel density estimation, we give a characterization of the kernels for which the parametric mean integrated squared error rate $n^{-1}$ may be obtained, where $n$ is the sample size. Also, for the cases where this rate is attainable, we give an asymptotic bandwidth choice that makes the kernel estimator consistent in mean integrated squared error at that rate and a numerical example showing the superior performance of the superkernel estimator when the bandwidth is properly chosen.
\end{abstract}

AMS Subject Class. (2000): Primary 62G05. Secondary 62G07.

Key words and phrases: optimal density estimation, convergence rates, mean integrated squared error, superkernel.

Short running title: Kernel density estimation at a parametric rate

Research supported by Spanish Ministerio de Ciencia y Tecnología project MTM2005-06348.

Proofs should be sent to: Agustín G. Nogales, Dpto. de Matemáticas, Universidad de Extremadura, Avda. de Elvas, s/n, 06071-Badajoz, SPAIN. e-mail: nogales@unex.es 


\section{Introduction}

If $X_{1}, \ldots, X_{n}$ is a sample from a probability distribution on the real line with density $f$, the kernel density estimator is given by

$$
f_{n, K, h}(x)=\frac{1}{n} \sum_{i=1}^{n} K_{h}\left(x-X_{i}\right)
$$

where the kernel $K$ is an integrable function with $\int K=1$, the bandwidth $h$ is a positive real number and we have used the notation $K_{h}(x)=K(x / h) / h$; see, e.g., Silverman (1986), Simonoff (1996) or Wand and Jones (1995). The $L_{2}$ error criterion will be used here; that is, we will measure the error of the estimate $f_{n, K, h}$ through the mean integrated square error (MISE), defined by

$$
\operatorname{MISE}_{f}\left(f_{n, K, h}\right)=E_{f} \int\left[f_{n, K, h}(x)-f(x)\right]^{2} d x .
$$

We will assume henceforth that all the kernels below are bounded functions, continuous at zero and such that $\int K^{2}<2 K(0)$. This technical conditions ensure that an optimal bandwidth $h_{0 n}(f)=\operatorname{argmin}_{h>0} \operatorname{MISE}_{f}\left(f_{n, K, h}\right)$ exists (see Chacón et al., 2006).

The main goal of this paper is to characterize the kernel functions that make the MISE converge to zero as fast as possible. Most commonly used kernels are the positive ones, because they produce bona fide density estimators; that is, estimators that, for every observed sample, provide a true density function (i.e., $f_{n, K, h} \geq 0$ and $\int f_{n, K, h}=1$ ). However, it is widely known that for positive kernels the MISE cannot decrease to zero faster than $n^{-4 / 5}$ (Rosenblatt, 1956). In this sense, some benefit can be obtained if we allow the kernel to take negative values (see Theorem 1 below), although the price to be paid is that the resulting estimate is not a positive function. Nevertheless, in a recent paper, Glad, Hjort and Ushakov (2003) show that, based on a non-bona fide estimator, it is possible to construct a bona fide one with even smaller MISE. Thus, there is no reason, in terms of MISE, to avoid the use of kernels taking negative values in density estimation. 
Watson and Leadbetter (1963) showed, in a very general background, that the MISE of kernel density estimators cannot decrease faster than $n^{-1}$. Davis (1977) characterized the class of densities for which this "parametric" rate $n^{-1}$ can be achieved (see Theorem 3 below). In this paper, we give a characterization of those kernels for which the MISE of the corresponding kernel estimator goes to zero at rate $n^{-1}$ for some density, so that together with the result of Davis (1977) we obtain a precise description of the family of densities and kernels for which the parametric rate is attainable (see Theorem 4). Besides, for this family we provide practical bandwidth-choice advice for achieving this rate.

\section{Main results}

Let us recall some facts about kernels. If we denote by $m_{j}(K)=\int x^{j} K(x) d x$ the $j$-th moment of a kernel $K$, we say that $K$ is of finite order if the set

$$
\mathcal{A}_{K}=\left\{j \in \mathbb{N}, j \geq 1: m_{j}(K) \neq 0\right\}
$$

is non-empty. In this case, $k=\min \mathcal{A}_{K}$ is called the order of the kernel $K$. If $\mathcal{A}_{K}=\varnothing$ then it is said that $K$ is a kernel of infinite order and such a kernel should satisfy $m_{j}(K)=0$ for $j=1,2, \ldots$

An example of an infinite order kernel is Natterer's kernel, whose characteristic function is given by $\varphi(t)=e^{-t^{2} /\left(1-t^{2}\right)} I_{[-1,1]}(t)$, where $I_{A}$ stands for the indicator function of the set $A$ (see Devroye and Lugosi, 2001, Ch. 17). If $K$ is the density of a symmetric distribution with finite variance, then $K$ is a kernel of order 2. A method for constructing a kernel of arbitrary finite order is shown in Schucany and Sommers (1977); however, if we want a kernel $K$ to have order $k>2$ then $K$ must necessarily take negative values.

Let us denote

$$
\Phi(n, f, K)=\min _{h>0} \operatorname{MISE}_{f}\left(f_{n, K, h}\right)
$$

that is, $\Phi(n, f, K)$ is the minimal MISE that can be achieved when we use the kernel $K$ and a sample of size $n$ to estimate $f$. The reason for using kernels 
of order greater than 2 (non-positive, therefore) rests upon the following theorem, which can be found, for instance, in Wand and Jones (1995).

Theorem 1. If $K \in L_{2}$ is a symmetric kernel of finite order $k$ and the density $f$ has a $k$-th continuous derivative belonging to $L_{2}$ then the minimal MISE that may be obtained by estimating $f$ using a kernel estimator with kernel $K$ is of exact order $n^{-2 k /(2 k+1)}$; that is,

$$
\lim _{n \rightarrow \infty} n^{2 k /(2 k+1)} \Phi(n, f, K)=\alpha_{1},
$$

where $\alpha_{1} \in(0, \infty)$ is a constant depending on $f$ and $K$.

Thus, as we make the kernel order grow, the rate of convergence of the optimal MISE to zero approaches the parametric rate $n^{-1}$, although the class of densities for which this rate is valid gets smaller and smaller. The question is: is there any kernel that effectively attains the rate $n^{-1}$ for some density? The kernels that achieve that MISE-rate for some density will deserve to be called superkernels; that is, a superkernel will be a kernel $K$ which satisfies,

$$
\lim _{n \rightarrow \infty} n \Phi(n, f, K)=\alpha_{2}
$$

for some density $f$, with $0<\alpha_{2}<\infty$. As stated in the previous section, our purpose here is to give a characterization of such superkernels.

In view of Theorem 1, one is tempted to conjecture that an infinite order kernel is a good candidate to be a superkernel; however, we will see below that an infinite order kernel does not need to be a superkernel.

Denote by $\varphi_{K}(t)$ the characteristic function of a kernel $K$ and

$$
\begin{aligned}
& S_{K}=\inf \left\{t \geq 0:\left|\varphi_{K}(t)-1\right| \neq 0\right\} \\
& T_{K}=\inf \left\{r \geq 0:\left|\varphi_{K}(t)-1\right| \neq 0 \text { a.e. for } t \geq r\right\} .
\end{aligned}
$$

That is, $S_{K}$ is the greatest value of $r$ such that $\varphi_{K}$ is identically equal to 1 on $[0, r]$ and $T_{K}$ is the greatest value of $t$ such that $\varphi_{K}(t)=1$. Notice that nearly every kernel used in practice satisfies $S_{K}=T_{K}$.

The next result gives a characterization of the class of superkernels, in terms of their characteristic functions. 
Theorem 2. Let $K$ be a kernel in $L_{2}$ such that $S_{K}=T_{K}$. The following statements are equivalent:

i) $S_{K}>0$.

ii) $\Phi(n, f, K)$ is of exact order $n^{-1}$ for some density $f \in L_{2}$.

The previous theorem allows us to give an alternative (and equivalent) definition of a superkernel: we will say that a kernel $K$ with $S_{K}=T_{K}$ is a superkernel if $S_{K}>0$; that is, if its characteristic function is identically equal to 1 in a neighborhood of the origin. This is just the classical definition of superkernel used in Devroye (1992) or in Glad, Hjort and Ushakov (2003), for instance. Thus, although this definition is not very intuitive, Theorem 2 allows us to conclude that it is just the one that we were looking for. Besides, from this characterization it follows that Natterer's kernel, which has infinite order, is not a superkernel; that is, the minimal MISE that we obtain using Natterer's kernel cannot decrease to zero at rate $n^{-1}$ for any density. A classical example of superkernel is given by the trapezoidal kernel $K(x)=(\cos x-\cos (2 x)) /\left(\pi x^{2}\right)$, which has characteristic function $\varphi_{K}(t)=$ $I_{[0,1)}(|t|)+(2-|t|) I_{[1,2)}(|t|)$, so that $S_{K}=T_{K}=1$; see Devroye and Lugosi (2001). Some more examples of superkernels are included in Section 3 of McMurry and Politis (2004), they are called infinite order flat-top kernels there.

The characterization of the class of densities for which the rate $n^{-1}$ is attainable is given in a paper by Davis (1977). Let us denote by $\varphi_{f}(t)$ the characteristic function of a density $f$ and

$$
\begin{aligned}
C_{f} & =\sup \left\{r \geq 0: \varphi_{f}(t) \neq 0 \text { a.e. for } t \in[0, r]\right\} \\
D_{f} & =\sup \left\{t \geq 0: \varphi_{f}(t) \neq 0\right\} .
\end{aligned}
$$

Notice that the support of $\varphi_{f}$ is contained in $\left(-D_{f}, D_{f}\right)$; moreover, this interval coincides with the support in the common case where $C_{f}=D_{f}$.

Theorem 3 (Davis, 1977). Let $f$ be a density in $L_{2}$. The following statements are equivalent: 
i) $D_{f}<\infty$; i.e., $\varphi_{f}$ has bounded support.

ii) $\Phi(n, f, K)$ is of exact order $n^{-1}$ for some kernel $K \in L_{2}$.

Davis' theorem states that in kernel density estimation the MISE may decrease to zero at rate $n^{-1}$ only if the characteristic function of the density we aim to estimate has bounded support. An example of this kind of density is given by the Fejér-de la Vallé-Poussin density, $f(x)=(1-\cos x) /\left(\pi x^{2}\right)$, which has characteristic function $\varphi_{f}(t)=(1-|t|) I_{[-1,1]}(t)$. Davis (1977) even provides a kernel estimator that achieves the parametric rate if the bandwidth is properly chosen (see also Ibragimov and Khasminskii, 1982); however, her estimator is based on the sinc function $S(x)=(\sin x) /\left(\pi x^{2}\right)$, which is not a kernel as it is not an integrable function. In contrast, our Theorem 2 is valid for true kernel functions and gives a condition that is not only sufficient but also necessary for kernel density estimation at a parametric rate.

We can combine theorems 2 and 3 to get:

Theorem 4. Let $K$ be a kernel with $S_{K}=T_{K}$ and $f$ a density, both in $L_{2}$. Then,

$$
\Phi(n, f, K) \text { is of exact order } n^{-1} \text { iff } S_{K}>0 \text { and } D_{f}<\infty \text {. }
$$

The theorem above gives a precise characterization of the only case where kernel density estimation at a parametric rate is possible. Then, we may wonder what would happen if we use a superkernel when the density does not fulfil the condition $D_{f}<0$, i.e., when kernel density estimation at a parametric rate is not possible. In the $L_{1}$ context, Devroye (1992) showed that superkernel estimators are rate-adaptive, in the sense that they achieve the best possible rate that the density permits. Below we show that this is also the case in the $L_{2}$ setup.

Theorem 5. Let $K$ be a superkernel and $f$ be a density, both in $L_{2}$. It is verified: 
i) (Smooth case) If $f$ has a $k$-th derivative in $L_{1} \cap L_{2}$, then $\Phi(n, f, K)$ goes to zero as $n^{-2 k /(2 k+1)}$ or faster; that is, the sequence

$$
n^{2 k /(2 k+1)} \Phi(n, f, K)
$$

is bounded.

ii) (Supersmooth case) If for some $\alpha>0$ and $\gamma>0$ the integral

$$
I_{\alpha, \gamma}(f)=\int e^{\gamma|t|^{\alpha}}\left|\varphi_{f}(t)\right|^{2} d t
$$

is finite, then $\Phi(n, f, K)$ goes to zero as $(\log n)^{1 / \alpha} / n$ or faster; that is, the sequence

$$
\frac{n}{(\log n)^{1 / \alpha}} \Phi(n, f, K)
$$

is bounded.

Remark 1. We have borrowed the terminology "smooth" and "supersmooth" case from Glad, Hjort and Ushakov (1999), where a result similar to our Theorem 5 is shown for the sinc kernel; see also Davis (1977). Notice that when $D_{f}<\infty$ we are in the supersmooth case for all $\alpha>0$. Some examples of densities with $I_{\alpha, \lambda}(f)<\infty$ include the standard Gaussian $(\alpha=2)$ and Cauchy $(\alpha=1)$ densities. Also, it should be remarked that Theorem 3.1 in Politis (2003) is the analogue to the previous result in a pointwise sense (rather than for the MISE criterion).

Remark 2. Denote $R(g)=\int g(x)^{2} d x$ for any $g \in L_{2}$. From the proof of Theorem 5 (see Section 4 below), in the smooth case the quantity $n^{2 k /(2 k+1)} \Phi(n, f, K)$ can be bounded by

$$
(2 k+1)(2 k)^{-2 k /(2 k+1)}\left(\frac{R(K)}{S_{K}}\right)^{2 k /(2 k+1)} R\left(f^{(k)}\right) .
$$

For all $k$, this bound depends on the superkernel $K$ only through $R(K) / S_{K}$; therefore, we could try to find the supernernel $K$ minimizing this value, as it is done in the finite-order case. For kernels of order 2, it is well-known that 
the kernel minimizing an asymptotic version of the MISE is the so-called Epanechinikov kernel; see, e.g., Silverman (1986). Here, in the superkernel case, we have $R(K) \geq S_{K} / \pi$ for all $K$. This lower bound is achievable if and only if $\varphi_{K}(t)=0$ for all $|t| \geq S_{K}$ but clearly, among all the superkernels satisfying such a condition, the only one fulfilling $R(K)=S_{K} / \pi$ is given by $\varphi_{K}(t)=I_{\left[-S_{K}, S_{K}\right]}(t)$, which corresponds to (a rescaled version of) the sinc kernel. In this sense, although the sinc function does not provide a proper kernel, it is the asymptotically optimal choice; that is, the analogue to the Epanechnikov kernel for the superkernel case.

Although Theorem 4 seems to be of purely theoretical interest, as it says nothing about the main problem in kernel density estimation, the choice of the bandwidth, this issue may be solved by using the next result, which can be found in Chacón et al. (2006). Let us recall the notation $h_{0 n}(f)$ for the $L_{2}$-optimal bandwidth; that is,

$$
h_{0 n}(f)=\underset{h>0}{\operatorname{argmin}} \operatorname{MISE}_{f}\left(f_{n, K, h}\right) .
$$

Theorem 6. Let $K$ be a kernel and $f$ a density, both in $L_{2}$. If $S_{K}=T_{K}$ or $C_{f}=D_{f}$ then

$$
h_{0 n}(f) \rightarrow S_{K} / D_{f} \text { as } n \rightarrow \infty .
$$

Moreover, if $S_{K}>0$ and $D_{f}<\infty$ then, for any fixed $h_{\star} \in\left(0, S_{K} / D_{f}\right]$ (not depending on $n$ ), we have

$$
E_{f}\left[f_{n, K, h_{\star}}(x)\right]=f(x), \text { for a.e. } x \in \mathbb{R}, \forall n \in \mathbb{N},
$$

so that $\operatorname{MISE}_{f}\left(f_{n, K, h_{\star}}\right)$ is of exact order $n^{-1}$.

Remark 3. Theorem 6 suggests taking $h=S_{K} / D_{f}$ under the conditions of Theorem 4. This is an asymptotic selection, as it is the limit of the optimal bandwidth sequence but, also, in this case it provides us with an unbiased kernel density estimator, whose MISE goes to zero at a parametric rate. Indeed, in such a situation we can bound

$$
n \Phi(n, f, K) \leq n \operatorname{MISE}_{f}\left(f_{n, K, S_{K} / D_{f}}\right) \leq D_{f} R(K) / S_{K},
$$


so that same argument as in Remark 1 shows that the sinc kernel is also the asymptotically optimal choice for the case where $D_{f}<\infty$.

Remark 4. Any bandwidth $h_{\star}$ as in the previous theorem may be called a global "zero-bias bandwidth". In a similar way, Sain and Scott (2002) show,

for non-negative kernels, the existence of local zero-bias bandwidths $h_{0}(x)$, not varying with $n$, for every $x$ in the region where $f$ is convex. Using this local bandwidths they also get a $n^{-1}$ rate, but with respect to the pointwise mean squared error.

\section{A numerical illustration}

Next we give a simple numerical example showing the performance of the superkernel estimators "at full power", that is, in the optimal situation where the characteristic function of the density has bounded support. To do so, we are going to focus on the aforementioned Fejér-de la Vallé-Poussin density

$$
f(x)=\frac{1-\cos x}{\pi x^{2}}, \quad x \in \mathbb{R},
$$

and the trapezoidal superkernel

$$
K(x)=\frac{\cos x-\cos (2 x)}{\pi x^{2}}, \quad x \in \mathbb{R} .
$$

For this superkernel, we will use two different bandwidth selection approaches: the first bandwidth is selected by a cross-validation method (see Silverman, 1986, or Wand and Jones, 1995); the second bandwidth comes from a version of the bandwidth selection procedure proposed by Politis (2003). This method aims to estimate $D_{f}$ making use of the empirical characteristic function, and it is closely related to the one proposed by Chiu (1991) for a similar problem in density estimation (see also Politis and Romano, 1999). If $\varphi_{n}(t)=n^{-1} \sum_{j=1}^{n} \exp \left\{i t X_{j}\right\}$ denotes the empirical characteristic function, $D_{f}$ is estimated by

$$
\widehat{D}_{n}=\inf \left\{D>0:\left|\varphi_{n}(D+t)\right|^{2}<c \frac{\log n}{n}, \forall t \in\left(0, \ell_{n}\right)\right\}
$$


where $c>0$ is a fixed constant and $\left(\ell_{n}\right)$ is a positive nondecreasing sequence. As suggested in Remark 3, the chosen bandwidth is then $\widehat{h}_{n}=1 / \widehat{D}_{n}$. Following the advice in Politis (2003), in all the simulations we have taken $c=1$ and $\ell_{n}=1$.

We want to compare this superkernel density estimator with the classical one, using a density function as a kernel. To this aim, we also include in the simulations the results for the Sheather-Jones method (Sheather and Jones, 1991), which uses the standard normal density as the kernel, so that it is known that the MISE cannot decrease faster than $n^{-4 / 5}$ (again, see Theorem 1 above).

We have tried these three methods for sample sizes $n=100$ (small), $n=400$ (medium) and $n=1600$ (large) over 100 simulated samples of each size drawn from the Fejér-de la Vallé-Poussin density. The results are shown in Table 1. For each estimator $\hat{f}_{n}$ and sample size we give the average and standard deviation of the 100 values of $\operatorname{ISE}\left(\hat{f}_{n}\right)=10^{3} \times \int\left(\hat{f}_{n}-f\right)^{2}$.

\begin{tabular}{|c|c|c|c|}
\hline $\mathrm{n}$ & ISE $_{\mathrm{CV}}$ & ISE $_{\mathrm{SJ}}$ & ISE $_{\mathrm{Pol}}$ \\
\hline \hline 100 & 3.36 & 3.04 & 2.53 \\
& $(4.38)$ & $(2.21)$ & $(2.28)$ \\
\hline 400 & 2.59 & 0.902 & 0.612 \\
& $(1.14)$ & $(0.549)$ & $(0.365)$ \\
\hline 1600 & 1.10 & 0.348 & 0.179 \\
& $(0.811)$ & $(0.172)$ & $(0.132)$ \\
\hline
\end{tabular}

Table 1: Simulation results for sample sizes $n=100,400,1600$. Averages and (standard deviations) of the ISE are given for each method.

As usual, it can be seen from Table 1 that the cross-validated selector is far more variable than the others. In this case, even the average ISE is also unacceptably large, when it is used together with a superkernel. In contrast, the selector of Politis does a good work: it is comparable with the SheatherJones method for small sample size, but the superior asymptotics of the superkernel estimator clearly begin to take their advantage yet for $n=400$. 
For large sample size, the better performance of the superkernel estimator is even more evident, obtaining nearly half the average ISE of the SheatherJones selector and less variance. Therefore, the usefulness of superkernels in density estimation becomes clear, at least in this case.

\section{Proofs}

The proof of our main result (Theorem 2) relies heavily on previous results that may be found in Chacón et al. (2006). For the sake of completeness we also include their statements here.

Lemma 1. Let $f$ be a density and $K$ a kernel, both in $L_{2}$. It is verified:

i) $R\left(K_{h} * f\right) \rightarrow R(f)$ as $h \rightarrow 0$.

ii) If $S_{K}=0$ then $h_{0 n}(f) \rightarrow 0$ as $n \rightarrow \infty$.

For the proof of Theorem 2 we will need an auxiliary result. It states that if we use a kernel $K$ with $S_{K}=0$, then the MISE-convergence rate is slower than $n^{-1}$ for every density. It can be applied, for instance, to finite-order kernels, as it is easy to show that any kernel of finite order satisfies $S_{K}=0$.

Lemma 2. If $K \in L_{2}$ is a kernel such that $S_{K}=0$ then, for every density $f \in L_{2}$, we have that

$$
\lim _{n \rightarrow \infty} n \Phi(n, f, K)=\infty .
$$

Proof. It is easy to show that

$$
\int \operatorname{Var}_{f}\left[f_{n, K, h}(x)\right] d x=R(K) /(n h)-R\left(K_{h} * f\right) / n,
$$

where $*$ stands for convolution (see Wand and Jones, 1995). Therefore,

$$
\begin{aligned}
n \Phi(n, f, K) & =n \operatorname{MISE}_{f}\left(f_{n, K, h_{0 n}(f)}\right) \\
& \geq n \int \operatorname{Var}_{f}\left[f_{n, K, h_{0 n}(f)}(x)\right] d x \\
& =\frac{R(K)}{h_{0 n}(f)}-R\left(K_{h_{0 n}(f)} * f\right)
\end{aligned}
$$

Then, the conclusion follows immediately from Lemma 1 . 
Proof of Theorem 2. If $S_{K}>0$, then Theorem 6 states that it suffices to consider a density with $D_{f}<\infty$, such as the Fejér-de la Vallée-Poussin density, to get a parametric MISE-convergence rate. On the other hand, the previous lemma shows precisely the implication $i i) \Rightarrow i$ ).

Proof of Theorem 5. In the smooth case, standard Fourier transform theory shows that the conditions on $f$ ensure that

$$
\int|t|^{2 k}\left|\varphi_{f}(t)\right|^{2} d t=2 \pi R\left(f^{(k)}\right)<\infty .
$$

Using Parseval identity, $2 \pi \operatorname{MISE}_{f}\left(f_{n, K, h}\right)=B(h)+V(h)$, where

$$
\begin{aligned}
& \left.0 \leq B(h)=\int\left|\varphi_{f}(t)\right|^{2} \mid \varphi_{K}(t h)-1\right)\left.\right|^{2} d t \\
& 0 \leq V(h)=\frac{1}{n h} \int\left|\varphi_{K}(t)\right|^{2} d t-\frac{1}{n} \int\left|\varphi_{f}(t)\right|^{2}\left|\varphi_{K}(t h)\right|^{2} d t .
\end{aligned}
$$

Then, we can bound $V(h)$ by $\int\left|\varphi_{K}\right|^{2} /(n h)$ and

$$
\begin{aligned}
B(h) & =\int_{|t|>S_{K} / h}\left|\varphi_{f}(t)\right|^{2}\left|\varphi_{K}(t h)-1\right|^{2} d t \\
& \leq \int_{|t|>S_{k} / h}\left|\varphi_{f}(t)\right|^{2} d t \\
& \leq \frac{h^{2 k}}{S_{K}^{2 k}} \int|t|^{2 k}\left|\varphi_{f}(t)\right|^{2} d t
\end{aligned}
$$

so that

$$
\operatorname{MISE}_{f}\left(f_{n, K, h}\right) \leq \frac{h^{2 k}}{S_{K}^{2 k}} R\left(f^{(k)}\right)+\frac{R(K)}{n h} .
$$

Calculating the minimum of the expression on the right-hand-side of the previous display, we get

$$
\Phi(n, f, K) \leq C n^{-2 k /(2 k+1)},
$$

as desired.

For the supersmooth case, the same kind of calculations can be used to bound

$$
B(h) \leq e^{-S_{K} \gamma / h^{\alpha}} I_{\alpha, \gamma}(f) .
$$

Now, taking $h$ to be of order $(\log n)^{-1 / \alpha}$ in $B(h)+V(h)$ gives the proof. 


\section{References}

Chacón, J. E., Montanero, J., Nogales, A. G. And Pérez, P. (2006).

On the existence and limit behavior of the optimal bandwidth in kernel density estimation. To appear in Statistica Sinica.

Chiu, S.-T. (1991) . Bandwidth selection for kernel density estimation. Annals of Statistics, 19, 1883-1905.

DAVIS, K. B. (1975). Mean square error properties of density estimates. Annals of Statistics, 3, 1025-1030.

Davis, K. B. (1977). Mean integrated square error properties of density estimates. Annals of Statistics, 5, 530-535.

Devroye, L. (1992). A note on the usefulness of superkernels in density estimation. Annals of Statistics, 20, 2037-2056.

Devroye, L. And Lugosi, G. (2001). Combinatorial Methods in Density Estimation. Springer-Verlag, New York.

Glad, I. K., Huort, N. L. And Ushakov, N. G. (1999). Upper bounds for the I-MSE and max-MSE of kernel density estimators. Statistical Research Report 18-99, Department of Mathematics, University of Oslo.

Glad, I. K., Huort, N. L. And Ushakov, N. G. (2003). Correction of density estimators that are not densities. Scandinavian Journal of Statistics, 30, 415-427.

Ibragimov, I. A. And Khasminskit, R. Z. (1982). Estimation of distribution density belonging to a class of entire functions. Theory of Probability and its Applications, 27, 551-562.

McMurry, T. L. And Politis, D. N. (2004). Nonparametric regression with infinite order flat-top kernels. Nonparametric Statistics, 16, $549-562$. 
Politis, D. N. (2003). Adaptive bandwidth choice. Nonparametric Statistics, 15, 517-533.

Politis, D. N. And Romano, J. P. (1999). Multivariate density estimation with general flat-top kernels of infinite order. Journal of Multivariate Analysis, 68, 1-25.

Schucany, W.R. And Sommers, J.P. (1977). Improvement of kerneltype density estimators. Journal of the American Statistical Association, 72, 420-423.

Rosenblatt, M. (1956). Remarks on some nonparametric estimates of a density function. Annals of Mathematical Statistics, 27, 832-837.

Sain, S. R. And Scott, D. W. (2002). Zero-bias locally adaptive density estimators. Scandinavian Journal of Statistics, 29, 441-460.

Sheather, S.J. And Jones, M.C. (1991). A reliable data-based bandwidth selection method for kernel density estimation. Journal of the Royal Statistical Society, Series B, 53, 683-690.

Silverman, B. W. (1986). Density Estimation for Statistics and Data Analysis. Chapman \& Hall, London.

Simonoff, J. S. (1996) . Smoothing Methods in Statistics. SpringerVerlag, New York.

Wand, M. P. And Jones, M. C. (1995). Kernel smoothing. Chapman \& Hall, London.

Watson, G. S. and Leabetter, M. R. (1963). On the estimation of the probability density, I. Annals of Mathematical Statistics, 34, 480491. 